\title{
Updating an LU factorization with Pivoting
}

\section{FLAME Working Note \#21}

\author{
Enrique S. Quintana-Ortí \\ Departamento de Ingeniería y Ciencia de Computadores \\ Universidad Jaume I \\ Campus Riu Sec \\ 12.071 Castellón, Spain \\ quintana@icc.uji.es \\ Robert A. van de Geijn \\ Department of Computer Sciences \\ The University of Texas at Austin \\ Austin, TX 78712 \\ rvdg@cs.utexas.edu
}

September 6, 2006

\begin{abstract}
We show how to compute an LU factorization of a matrix when the factors of a leading principle submatrix are already known. The approach incorporates pivoting akin to partial pivoting, a strategy we call incremental pivoting. An implementation using the Formal Linear Algebra Methods Environment (FLAME) Application Programming Interface (API) is described. Experimental results demonstrate practical numerical stability and high performance on an Intel Itanium2 processor based server.
\end{abstract}

\section{Introduction}

In this paper we consider the $\mathrm{LU}$ factorization of a nonsymmetric matrix, $A$, partitioned as

$$
A \rightarrow\left(\begin{array}{c|c}
B & C \\
\hline D & E
\end{array}\right)
$$

when a factorization of $B$ is to be reused as the other parts of the matrix change. This is known as the updating of an LU factorization.

Applications arising in Boundary Element Methods (BEM) often lead to very large dense linear systems [3, 5]. For many of these applications the goal is to optimize a feature of an object. For example, BEM may be used to model the radar signature of an airplane. In an effort to minimize this signature, it may be necessary to optimize the shape of a certain component of the airplane. If the degrees of freedom associated with this component are ordered last among all degrees of freedom, the matrix presents the structure given in (1). Now, as the shape of the component is modified, it is only the matrices $C, D$, and $E$ that change together with the right-hand side vector of the corresponding linear system. Since the dimension of $B$ is frequently much larger than those of the remaining three matrices, it is desirable to factorize $B$ only once and to update the factorization as $C, D$, and $E$ change. A standard LU factorization with partial pivoting does not provide a convenient solution to this problem, since the rows to be swapped during the application of the permutations may not lie only within $B$. 
Little literature exists on this important topic. We have been made aware that an unblocked OOC algorithm similar to our algorithm was reported in [22], but we have not been able to locate a copy of that report. The proposed addition of this functionality to LAPACK is discussed in [4]. We already discussed preliminary results regarding the algorithm proposed in the current paper in a conference paper [12], in which its application to out-of-core LU factorization with pivoting is the main focus ${ }^{1}$. In [9] the updating of a QR factorization via techniques that are closely related to those proposed for the LU factorization in the current paper is reported.

The paper is organized as follows: in Section 2 we review algorithms for computing the LU factorization with partial pivoting. In Section 3, we discuss how to update an LU factorization by considering the factorization of a $2 \times 2$ blocked matrix. The key insight of the paper is found in this section: Highperformance blocked algorithms can be synthesized by combining the pivoting strategies of LINPACK and LAPACK. Numerical stability is discussed in Section 4 and performance is reported in Section 5. Concluding remarks are given in the final section. We hereafter assume that the reader is familiar with Gauss transforms, their properties, and how they are used to factor a matrix.

We start indexing elements of vectors and matrices at 0. Capital letters, lower case letter, and lower case Greek letters will be used to denote matrices, vectors, and scalars, respectively. The identity matrix of order $n$ is denoted by $I_{n}$.

\section{The LU factorization with partial pivoting}

Given an $n \times n$ matrix $A$, its LU factorization with partial pivoting is given by $P A=L U$. Here $P$ is a permutation matrix of order $n, L$ is $n \times n$ unit lower triangular, and $U$ is $n \times n$ upper triangular. We will denote the computation of $P, L$, and $U$ by

$$
[A, p]:=[\{L \backslash U\}, p]=\mathrm{LU}(A),
$$

where $\{L \backslash U\}$ is the matrix whose strictly lower and upper triangular parts equal those of $L$ and $U$, respectively. Matrix $L$ has ones on the diagonal, which need not be stored, and the factors $L$ and $U$ overwrite the original contents of $A$. The permutation matrix is generally stored in a vector $p$ of $n$ integers.

Solving the linear system $A x=b$ now becomes a matter of solving $L y=P b$ followed by $U x=y$. These two stages are referred to as forward substitution and backward substitution, respectively.

\subsection{Unblocked right-looking LU factorization}

Two unblocked algorithms for computing the LU factorization with partial pivoting are given in Figure 1. There, $n(\cdot)$ stands for the number of columns of a matrix; the thick lines in the matrices/vectors denote how far computation has progressed; $\operatorname{Pivot}(x)$ determines the element in $x$ with largest magnitude, swaps that element with the top element, and returns the index of the element that was swapped; and $P\left(\pi_{1}\right)$ is the permutation matrix constructed by interchanging row 0 and row $\pi_{1}$ of the identity matrix. The dimension of a permutation matrix will not be specified since it is obvious from the context in which it is used. We believe the rest of the notation to be intuitive $[2,1]$. Both algorithms correspond to what is usually known as the right-looking variant. Upon completion matrices $L$ and $U$ overwrite $A$.

The LINPACK variant, $L U_{\text {UNB }}^{\mathrm{LIN}}$ hereafter, computes the LU factorization as a sequence of Gauss transforms interleaved with pivot matrices:

$$
L_{n-1}\left(\begin{array}{c|c}
I_{n-1} & 0 \\
\hline 0 & P\left(\pi_{n-1}\right)
\end{array}\right) \cdots L_{1}\left(\begin{array}{c|c}
1 & 0 \\
\hline 0 & P\left(\pi_{1}\right)
\end{array}\right) L_{0} P\left(\pi_{0}\right) A=U .
$$

For the LAPACK variant, $\mathrm{LU}_{\mathrm{UNB}}^{\mathrm{LAP}}$, it is recognized that by swapping those rows of matrix $L$ that were already computed and stored to the left of the column that is currently being eliminated, the order of

\footnotetext{
${ }^{1}$ More practical approaches to out-of-core LU factorization with partial pivoting exist [19, 18, 15], which is why that application of the approach is not further mentioned.
} 


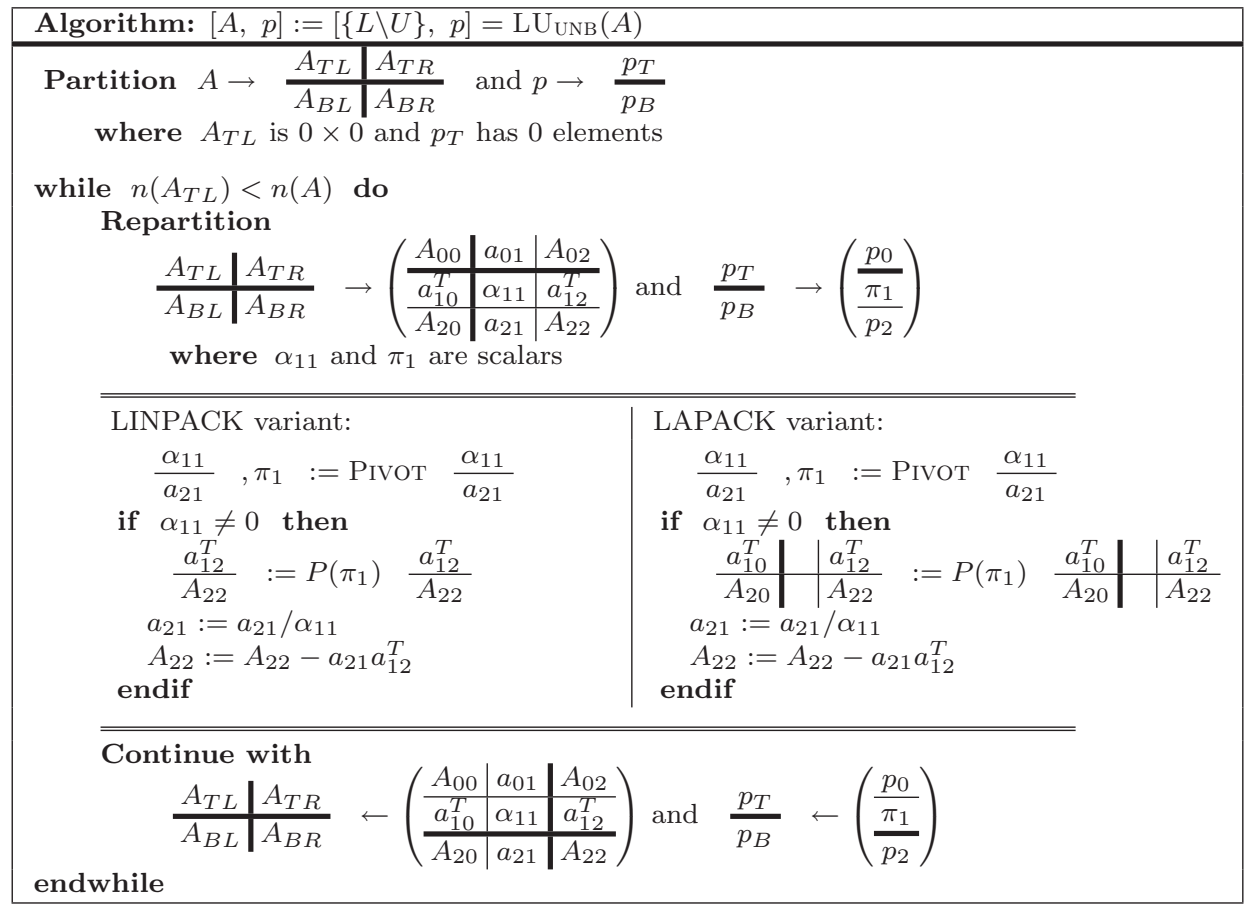

Figure 1: LINPACK and LAPACK unblocked algorithms for the LU factorization.

the Gauss transforms and the pivot matrices can be rearranged so that $P(p) A=L U$. Here $P(p)$, with $p=\left(\pi_{0}|\cdots| \pi_{n-1}\right)^{T}$, denotes the $n \times n$ permutation

$$
\left(\begin{array}{c|c}
I_{n-1} & 0 \\
\hline 0 & P\left(\pi_{n-1}\right)
\end{array}\right) \cdots\left(\begin{array}{c|c}
1 & 0 \\
\hline 0 & P\left(\pi_{1}\right)
\end{array}\right) P\left(\pi_{0}\right) .
$$

Both algorithms will execute to completion even if an exact zero is encountered on the diagonal of $U$. This is important since it is possible that matrix $B$ in (1) is singular even if $A$ is not.

The difference between the two algorithms becomes most obvious when forward substitution is performed. For the LINPACK variant forward substitution requires the application of permutations and Gauss transforms to be interleaved. For the LAPACK algorithm, the permutations can be applied first, after which a clean lower triangular solve yields the desired (intermediate) result: $L y=P(p) b$. Depending on whether the LINPACK or the LAPACK variant was used for the LU factorization, we denote the forward substitution stage respectively by $y:=\mathrm{FS}^{\mathrm{LIN}}(A, p, b)$ or $y:=\mathrm{FS}^{\mathrm{LAP}}(A, p, b)$, where $A$ and $p$ are assumed to be the outputs of the corresponding factorization.

\subsection{Blocked right-looking LU factorization}

It is well-known that high performance can be achieved in a portable fashion by casting algorithms in terms of matrix-matrix multiplication $[13,10,14,8]$. In Figure 2 we show LINPACK(-like) and LAPACK blocked algorithms, $\mathrm{LU}_{\mathrm{BLK}}^{\mathrm{LIN}}$ and $\mathrm{LU} \mathrm{BLK}_{\mathrm{BL}}^{\mathrm{LAP}}$ respectively, both built upon an LAPACK unblocked algorithm. The former algorithm really combines the LAPACK style of pivoting, within the factorization of a panel of width $b$, with the LINPACK style of pivoting. The two algorithms attain high performance on modern architectures with (multiple levels of) cache memory by casting the bulk of the computation in terms of the matrixmatrix multiplication $A_{22}:=A_{22}-L_{21} U_{12}$, also called a rank-k update, which is known to achieve high performance $[6]$. 


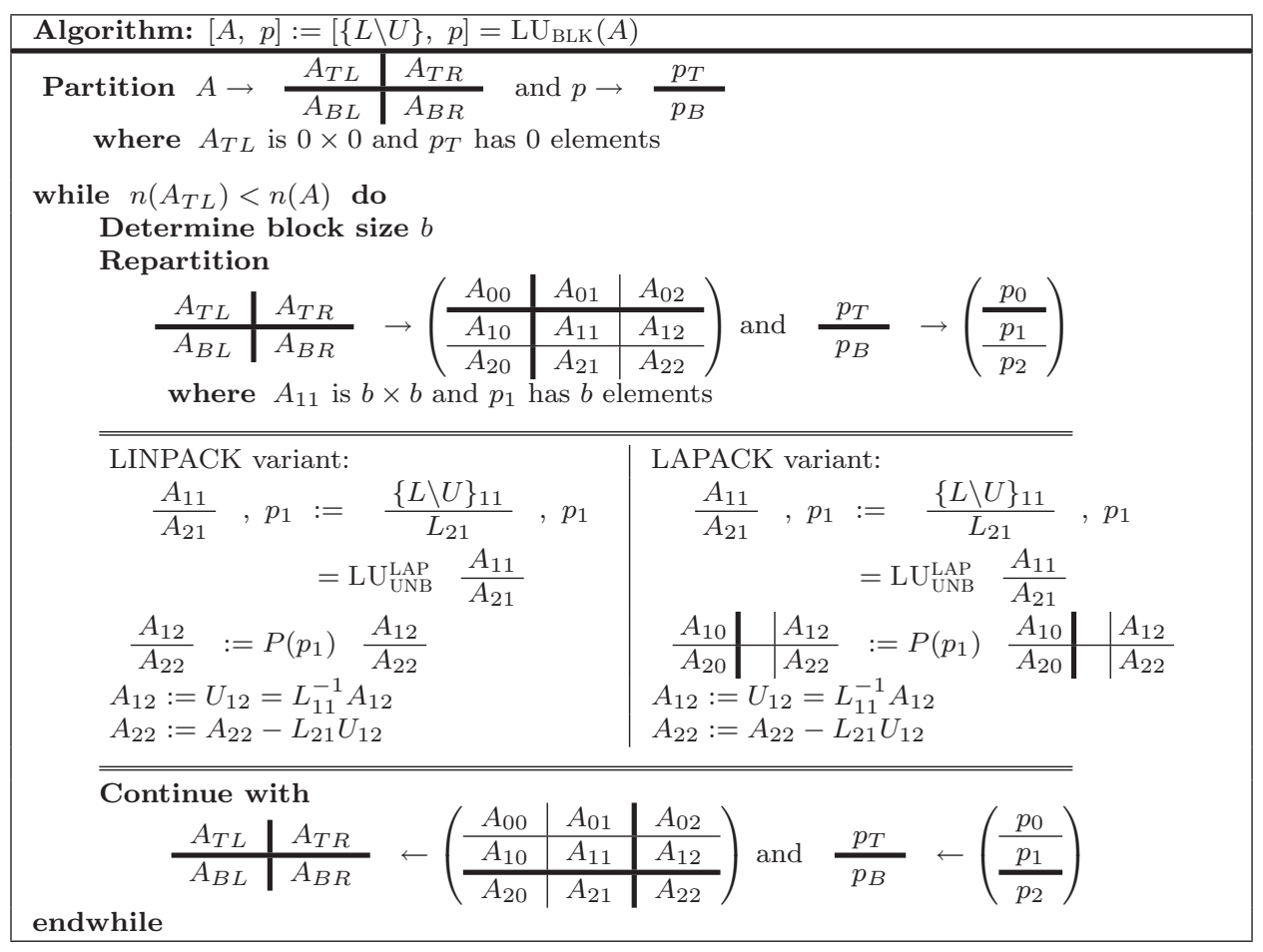

Figure 2: LINPACK and LAPACK blocked algorithms for the LU factorization built upon an LAPACK unblocked factorization.

\section{Updating an LU factorization}

In this section we discuss how to compute the LU factorization of the matrix in (1) in such a way that the LU factorization with partial pivoting of $B$ can be reused if $D, C$, and $E$ change. We consider $A$ in (1) to be of dimension $n \times n$, with square $B$ and $E$ of orders $n_{B}$ and $n_{E}$, respectively. For reference, factoring the matrix in (1) using the standard LU factorization with partial pivoting costs $\frac{2}{3} n^{3}$ flops (floating-point arithmetic operations). In this expression (and future computational cost estimates) we neglect insignificant terms of lower-order complexity, including the cost of pivoting the rows.

\subsection{Basic procedure}

We propose employing the following procedure, consisting of 5 steps, which computes an $L U$ factorization with incremental pivoting of the matrix in (1):

Step 1: Factor $B$. Compute the LU factorization with partial pivoting

$$
[B, p]:=[\{L \backslash U\}, p]=\mathrm{LU}_{\mathrm{BLK}}^{\mathrm{LAP}}(B) .
$$

This step is skipped if $B$ was already factored. If the factors are to be used for future updates to $C, D$, and $E$, then $U$ needs to be saved since it is overwritten by subsequent steps.

Step 2: Update $C$ consistent with the factorization of $B$ :

$$
C:=\operatorname{FS}^{\mathrm{LAP}}(B, p, C)
$$




\begin{tabular}{|l|c|c|c|}
\hline \multirow{2}{*}{ Operation } & \multicolumn{3}{|c|}{ Approximate cost (in flops) } \\
\cline { 2 - 4 } & $\begin{array}{c}\text { Basic } \\
\text { procedure }\end{array}$ & $\begin{array}{c}\text { SA LAPACK } \\
\text { procedure }\end{array}$ & $\begin{array}{c}\text { SA LINPACK } \\
\text { procedure }\end{array}$ \\
\hline 1: Factor $B$ & $\frac{2}{3} n_{B}^{3}$ & $\frac{2}{3} n_{B}^{3}$ & $\frac{2}{3} n_{B}^{3}$ \\
\hline 2: Update $C$ & $n_{B}^{2} n_{E}$ & $n_{B}^{2} n_{E}$ & $n_{B}^{2} n_{E}$ \\
\hline 3: Factor $\left(\frac{U}{D}\right)$ & $n_{B}^{2} n_{E}+\frac{2}{3} n_{B}^{3}$ & $n_{B}^{2} n_{E}+\frac{1}{2} b n_{B}^{2}$ & $n_{B}^{2} n_{E}+\frac{1}{2} b n_{B}^{2}$ \\
\hline 4: Update $\left(\frac{C}{E}\right)$ & $2 n_{B} n_{E}^{2}+n_{B}^{2} n_{E}$ & $2 n_{B} n_{E}^{2}+n_{B}^{2} n_{E}$ & $2 n_{B} n_{E}^{2}+b n_{B} n_{E}$ \\
\hline 5: Factor $E$ & $\frac{2}{3} n_{E}^{3}$ & $\frac{2}{3} n_{E}^{3}$ & $\frac{2}{3} n_{E}^{3}$ \\
\hline Total & $\frac{2}{3} n^{3}+\frac{2}{3} n_{B}^{3}+n_{B}^{2} n_{E}$ & $\frac{2}{3} n^{3}+n_{B}^{2}\left(\frac{1}{2} b+n_{E}\right)$ & $\frac{2}{3} n^{3}+b n_{B}\left(\frac{n_{B}}{2}+n_{E}\right)$ \\
\hline
\end{tabular}

Table 1: Computational cost (in flops) of the different approaches to compute the LU factorization of the matrix in (1). The highlighted costs are those incurred in excess of the cost of a standard LU factorization.

Step 3: Factor $\left(\frac{U}{D}\right)$. Compute the LU factorization with partial pivoting

$$
\left[\left(\frac{\{\bar{L} \backslash \bar{U}\}}{D}\right), r\right]:=\operatorname{LU}_{\mathrm{BLK}}^{\mathrm{LIN}}\left(\frac{U}{D}\right) .
$$

Here $\bar{U}$ overwrites the upper triangular part of $B$ (where $U$ was stored before this operation). The lower triangular matrix $\bar{L}$ that results needs to be stored separately, since both $L$, computed in Step 1 , and $\bar{L}$ are needed during the forward substitution stage when solving a linear system.

Step 4: Update $\left(\frac{C}{E}\right)$ consistent with the factorization of $\left(\frac{U}{D}\right)$ :

$$
\left(\frac{C}{E}\right):=\mathrm{FS}^{\mathrm{LIN}}\left(\left(\frac{\bar{L}}{D}\right), r,\left(\frac{C}{E}\right)\right) .
$$

Step 5: Factor E. Finally, compute the LU factorization with partial pivoting

$$
[E, s]:=\operatorname{LU}_{\mathrm{BLK}}^{\mathrm{LAP}}(E) .
$$

\subsection{Analysis of the basic procedure}

For now, the factorization in Step 3 does not take advantage of any zeroes below the diagonal of $U$ : After matrix $B$ is factored and $C$ is updated, the matrix $\left(\begin{array}{c|c}U & C \\ \hline D & E\end{array}\right)$ is factored as if it is a matrix without special structure. Its cost is stated in the column labeled "Basic procedure" in Table 1. If $n_{E}$ is small (that is, $n_{B} \approx n$ ), there is clearly no benefit to reusing an already factored $B$. Also, the procedure requires additional storage for the $n_{B} \times n_{B}$ lower triangular matrix $\bar{L}$ computed in Step 3 .

We describe next how to reduce both the computational and storage requirements by exploiting the upper triangular structure of $U$ during Steps 3 and 4 .

\subsection{Exploiting the structure in Step 3}

A blocked algorithm that exploits the upper triangular structure of $U$ is given in Figure 3 and illustrated in Figure 4. We name this algorithm $\mathrm{LU}_{\mathrm{BLK}}^{\mathrm{SA}-\mathrm{LIN}}$ to reflect that it computes a "Structure-Aware" (SA) LU factorization. At each iteration of the algorithm, the panel of $b$ columns consisting of $\left(\frac{U_{11}}{D_{1}}\right)$ is factored using the LAPACK blocked algorithm LU UNB . (In our implementation this algorithm is modified to, in 


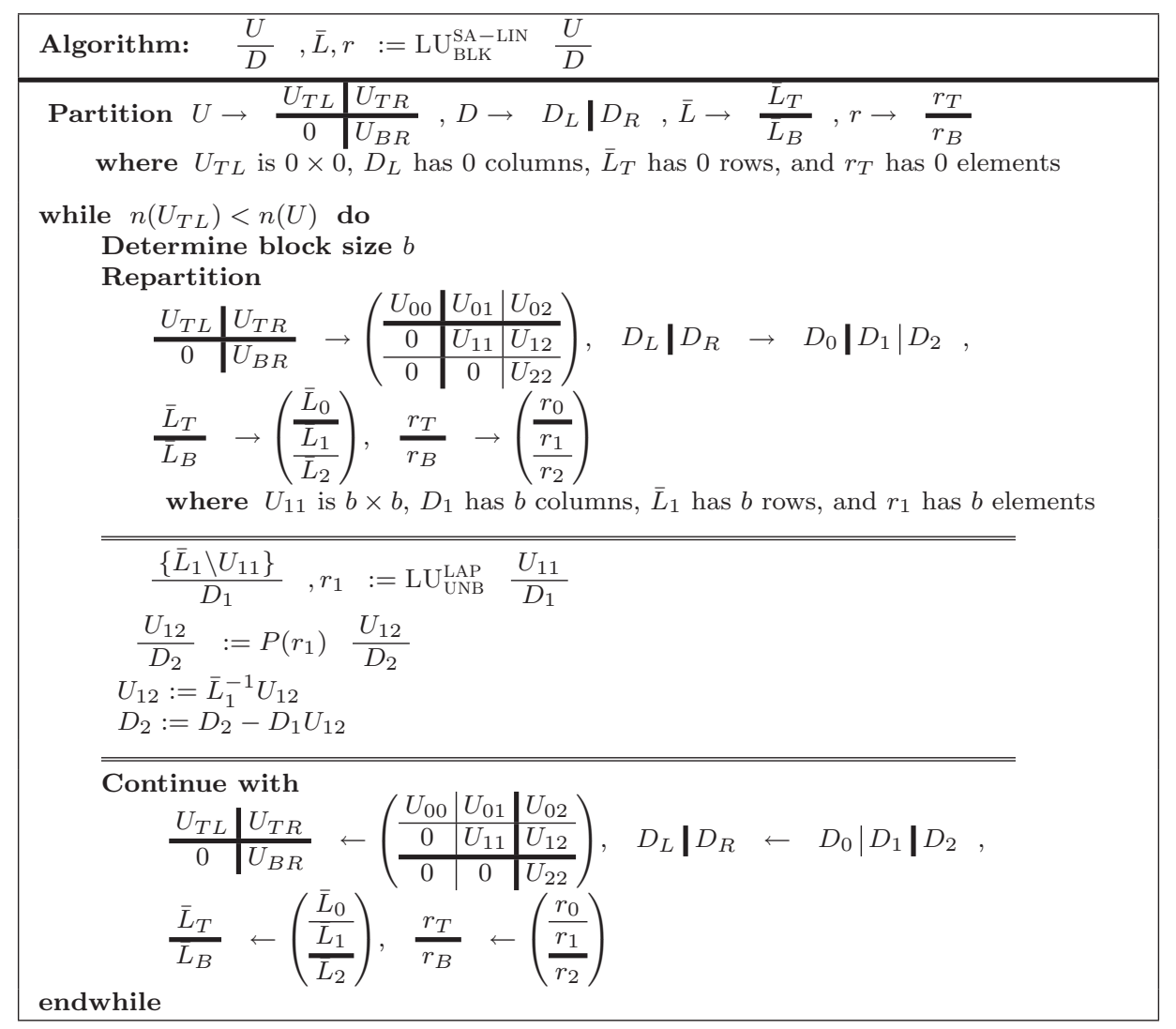

Figure 3: SA-LINPACK blocked algorithm for the LU factorization of $\left(U^{T}, D^{T}\right)^{T}$ built upon an LAPACK blocked factorization.

addition, take advantage of the zeroes below the diagonal of $U_{11}$.) As part of the factorization, $U_{11}$ is overwritten by $\left\{\bar{L}_{1} \backslash \bar{U}_{11}\right\}$. However, in order to preserve the strictly lower triangular part of $U_{11}$ (where part of the matrix $L$, that was computed in Step 1, is stored), we employ the $b \times b$ submatrix $\bar{L}_{1}$ of the $n_{B} \times b$ array $\bar{L}$. As in the LINPACK blocked algorithm in Figure 2, the LAPACK and LINPACK styles of pivoting are combined: the current panel of columns are pivoted using the LAPACK approach but the pivots from this factorization are only applied to $\left(\frac{U_{12}}{D_{2}}\right)$.

The cost of this approach is given in Step 3 of the column labeled "SA LINPACK procedure" in Table 1. The extra cost comes from the updates of $U_{12}$ in Figure 3 which, provided $b \ll n_{B}$, is insignificant compared to $\frac{2}{3} n^{3}$.

An SA LAPACK blocked algorithm for Step 3 only differs from that in Figure 3 in that, at a certain iteration, after the LU factorization of the current panel is computed, the pivots have to be applied to $\left(\frac{U_{10}}{D_{0}}\right)$ as well. As indicated in Step 3 of the column labeled "SA LAPACK procedure", this does not incur extra cost for this step. However, it does require an $n_{B} \times n_{B}$ array for storing $\bar{L}$ (see Figure 4 ) and, as we will see next, makes Step 4 more expensive.

\subsection{Revisiting the update in Step 4}

The same optimizations made in Step 3 must now be carried over to the update of $\left(\frac{C}{E}\right)$. The algorithm for this is given in Figure 5. Computation corresponding to zeroes is avoided so that the cost of performing 

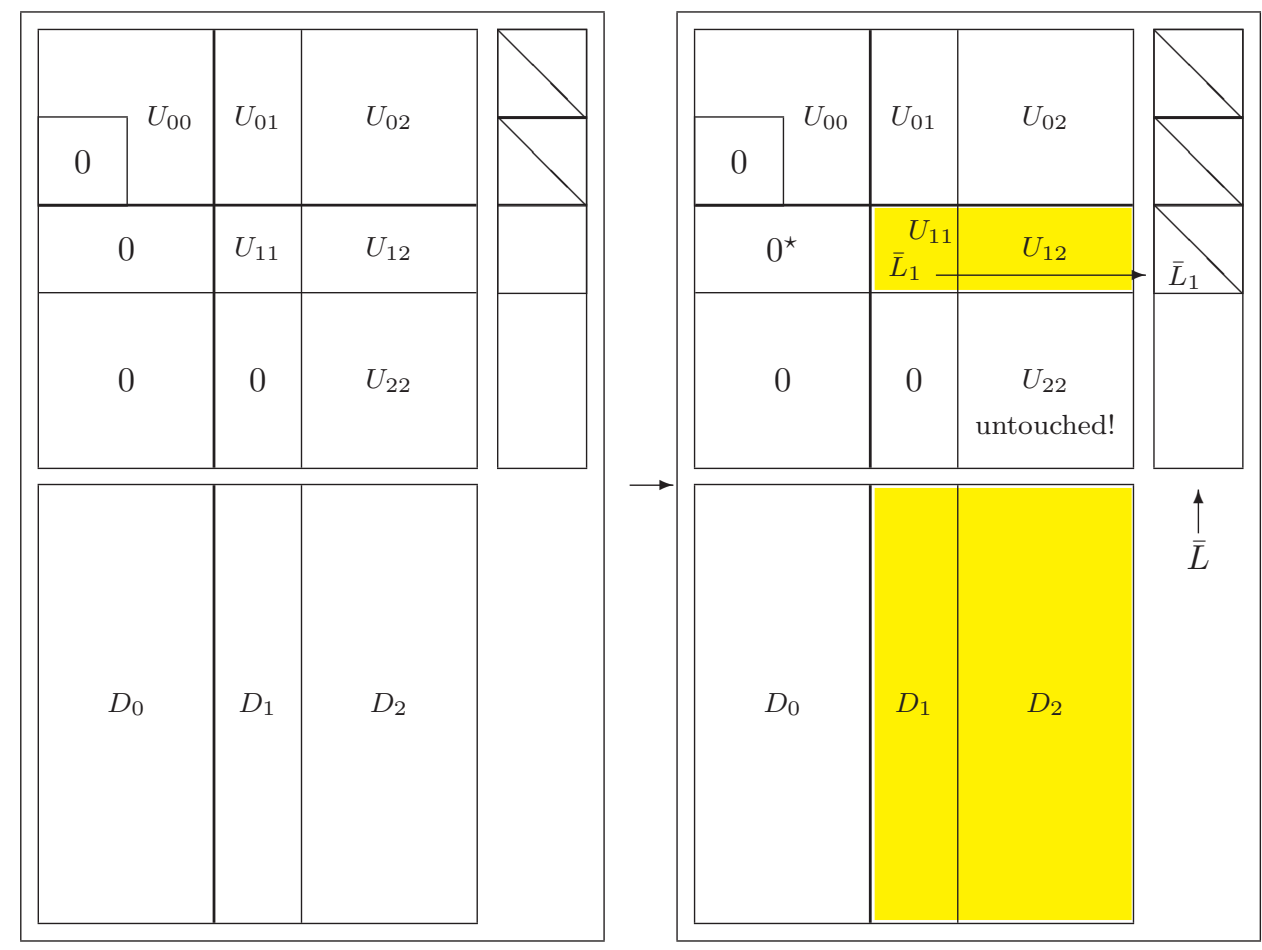

Figure 4: Illustration of an iteration of the SA LINPACK blocked algorithm used in Step 3 and how it preserves most of the zeroes in $U$. The zeroes below the diagonal are preserved, except within the $b \times b$ diagonal blocks, where pivoting will fill below the diagonal. The shaded areas are the ones updated as part of the current iteration. The fact that $U_{22}$ is not updated demonstrates how computation can be reduced. If the SA LAPACK blocked algorithm was used, then nonzeroes would appear during this iteration in the block marked as $0^{\star}$, due to pivoting; as a result, upon completion, zeros would be lost in the full strictly lower triangular part of $U$.

the update is $2 n_{B} n_{E}^{2}+b n_{B} n_{E}$ flops, as indicated in Step 4 of Table 1.

Applying the SA LAPACK blocked algorithm in Step 3 destroys the structure of the lower triangular matrix, which cannot be recovered during the forward substitution stage in Step 4, and explains the additional cost reported for this variant in Table 1.

\subsection{Key contribution}

The difference in cost of the three different approaches analyzed in Table 1 is illustrated in Figure 6 . It reports the ratios between the costs of the different procedures described above and that of the LU factorization with partial pivoting for a matrix with $n_{B}=1000$ and different values of $n_{E}$ using $b=32$. The analysis shows that the overhead of the SA LINPACK procedure is consistently low. On the other hand, as $n_{E} / n \rightarrow 1$ the cost of the basic procedure, which is initially twice as expensive as that of the LU factorization with partial pivoting, is decreased. The SA LAPACK procedure only presents a negligible overhead when $n_{E} \rightarrow 0$ that is, when the dimension of the update is very small.

The key insight of the proposed approach is the recognition that combining LINPACK- and LAPACKstyle pivoting allows one to use a blocked algorithm while avoiding filling most of the zeroes in the lower triangular part of $U$. This, in turn, makes the extra cost of Step 4 acceptable. In other words, for the SA LINPACK procedure, the benefit of the higher performance of the blocked algorithm comes at the expense of a lower-order amount of extra computation. 


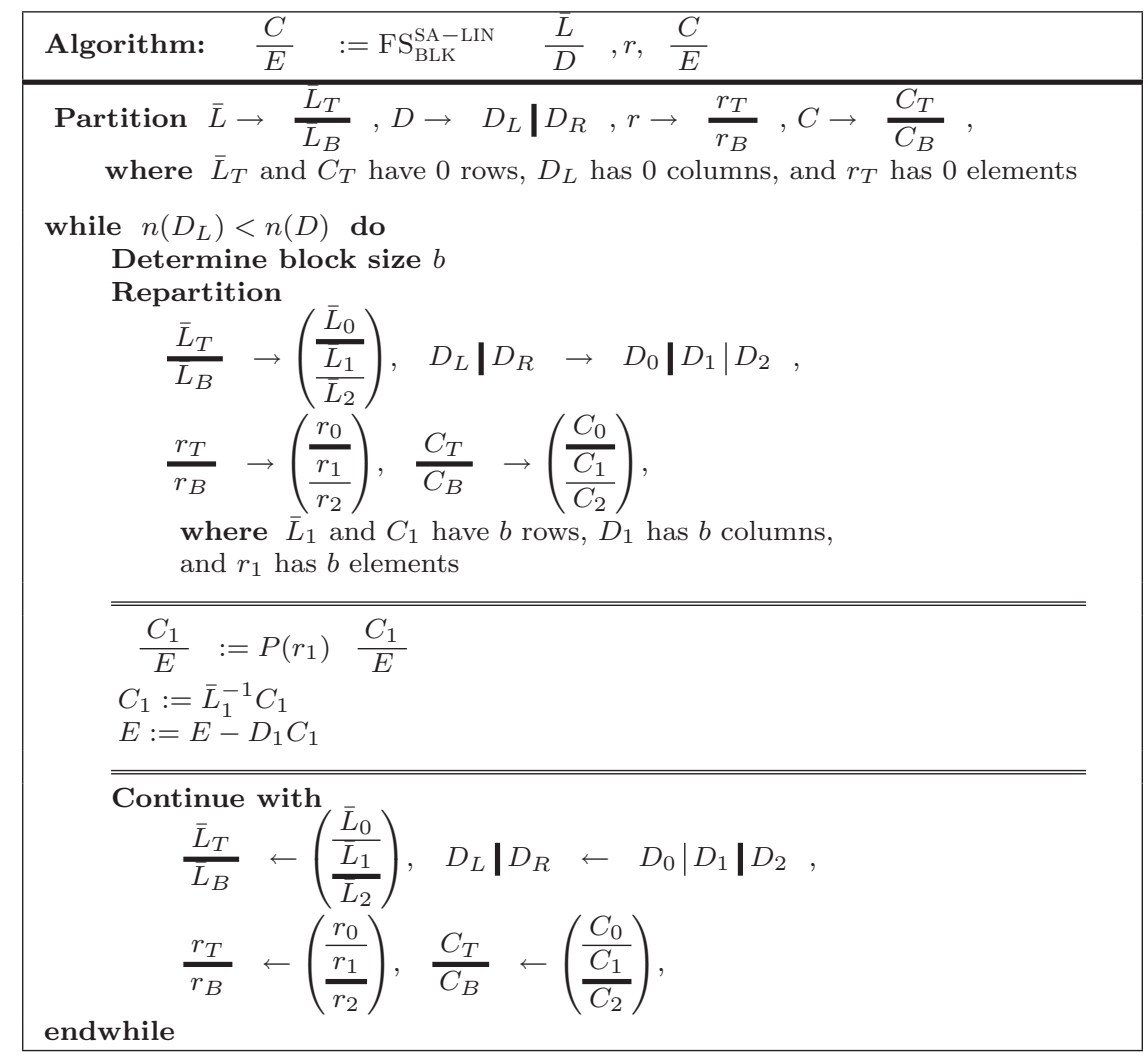

Figure 5: SA-LINPACK blocked algorithm for the update of $\left(C^{T}, E^{T}\right)^{T}$ consistent with the SA-LINPACK blocked LU factorization of $\left(U^{T}, D^{T}\right)^{T}$.

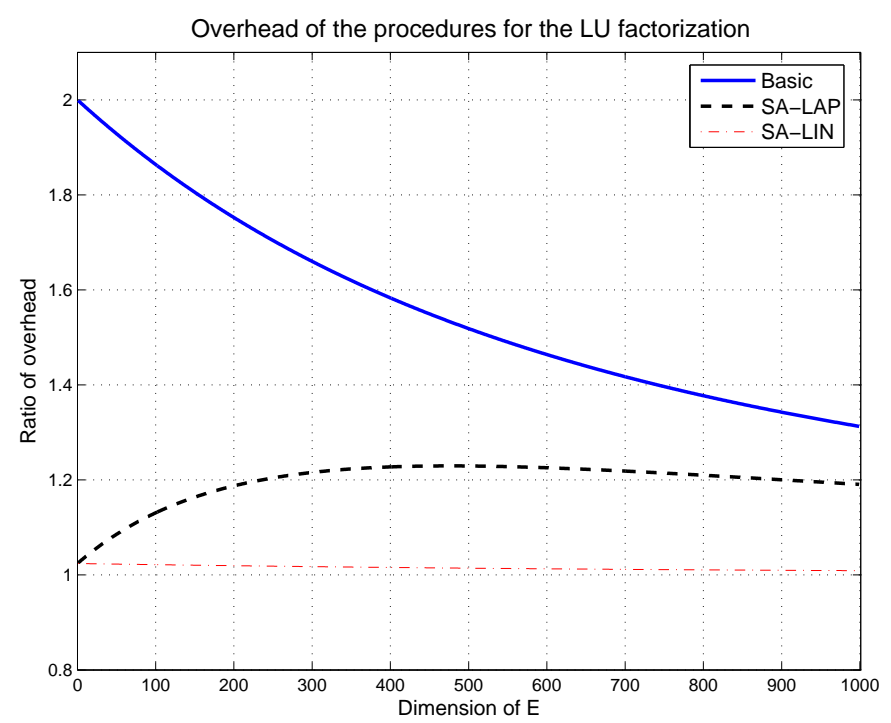

Figure 6: Overhead cost of the different approaches to compute the the LU factorization in (1) with respect to the cost of the LU factorization with partial pivoting. 


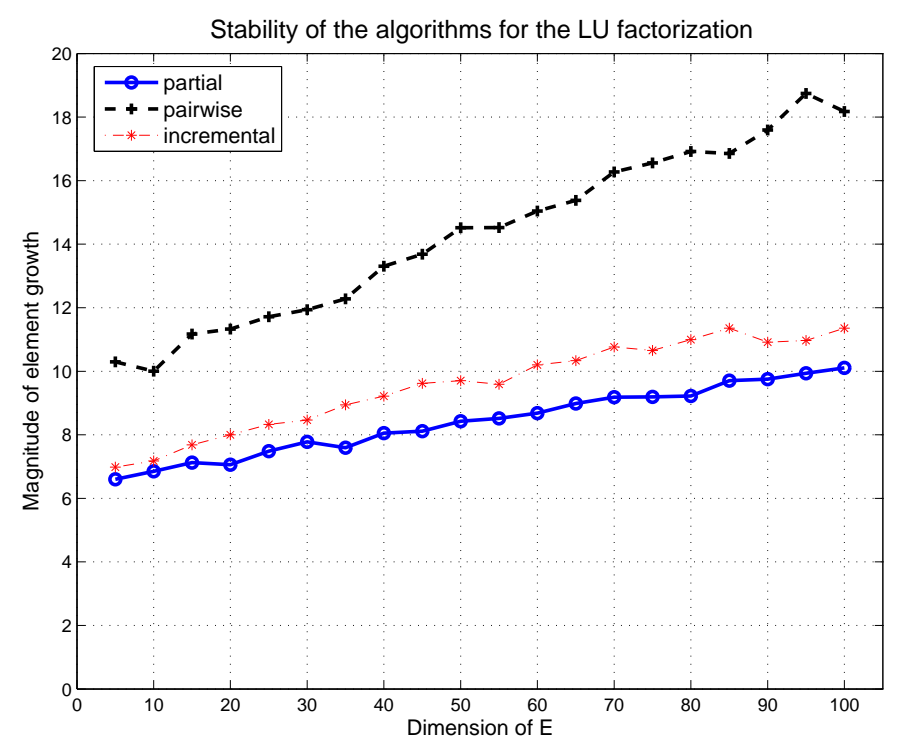

Figure 7: Element growth in the LU factorization using different pivoting techniques.

\section{Remarks on Numerical Stability}

The algorithm for the LU factorization with incremental pivoting carries out a sequence of row permutations (corresponding to the application of pivots) which are different from those that would be performed in an LU factorization with partial pivoting. Therefore, the numerical stability of this algorithm is also different. In this section we provide some remarks on the stability of the new algorithm. We note that all three procedures described in the previous section (basic, SA LINPACK, and SA LAPACK) perform the same sequence of row permutations.

The numerical (backward) stability of an algorithm that computes the LU factorization of a matrix $A$ depends on the growth factor [17]

$$
\rho=\frac{\|L\|\|U\|}{\|A\|},
$$

which is basically determined by the problem size and the pivoting strategy. For example, the growth factors of complete, partial, and pairwise ([21, p. 236]) pivoting have been demonstrated to be bounded as $\rho_{c} \leq n^{1 / 2}\left(2 \cdot 3^{1 / 2} \cdots n^{1 / n-1}\right), \rho_{p} \leq 2^{n-1}$, and $\rho_{w} \leq 4^{n-1}$, respectively [16, 17]. Statistical models and extensive experimentations in [20] showed that, on average, $\rho_{c} \approx n^{1 / 2}, \rho_{p} \approx n^{2 / 3}$, and $\rho_{w} \approx n$, inferring that in practice partial/pairwise pivoting are both numerically stable, and pairwise pivoting can be expected to numerically behave only slightly worse than partial pivoting.

The new algorithm applies partial pivoting during the factorization of $B$ and then again in the factorization of $\left(\frac{U}{D}\right)$. This can be considered as a blocked variant of pairwise pivoting. Thus, we can expect an element growth for the algorithm that is between those of partial and pairwise pivoting. Next we elaborate an experiment that provides evidence in support of this observation.

In Figure 7 we report the element growths observed during the computation of the LU factorization of matrices as in (1), with $n_{B}=100$ and dimensions for $E$ ranging from $n_{E}=5$ to 100 using partial, incremental, and pairwise pivoting. The entries of the matrices are generated randomly, chosen from a uniform distribution in the interval $(0.0,1.0)$. The experiment was carried out on an Intel Xeon processor using MATLAB@ 7.0.4 (IEEE double-precision arithmetic). The results report the average element growth for 100 different matrices for each matrix dimension. The figure shows that the growth factor of incremental pivoting is smaller than that of pairwise pivoting and approximates that of partial pivoting. 
For those who are not sufficiently satisfied with the element growth of incremental pivoting, we propose to perform a few refinement iterations of the solution to $A x=b$ as this guarantees stability at a low computational cost [11].

\section{Performance}

In this section we report results for a high-performance implementation of the SA LINPACK procedure.

\subsection{Implementation}

The FLAME library (Version 0.9) was used to implement a high-performance LU factorization with partial pivoting and the SA LINPACK procedure. The benefit of this API is that the code closely resembles the algorithms as they are presented in Figures 1-3 and 5. The performance of the FLAME LU factorization with partial pivoting is highly competitive with LAPACK and vendor implementations of this operation.

The implementations can be examined by visiting

$$
\text { http://www.cs.utexas.edu/users/flame/Publications/ }
$$

\subsection{Platform}

Performance experiments were performed in double-precision arithmetic on a Intel Itanium2 (1.5 GHz) processor based workstation capable of attaining 6 GFLOPS ( $10^{9}$ flops per second). For reference, the algorithm for the FLAME LU factorization with partial pivoting delivered 4.8 GFLOPS for a $2000 \times 2000$ matrix. The implementation was linked to the GotoBLAS R1.6 Basic Linear Algebra Subprograms (BLAS) library [7]. The BLAS routine DGEMm which is used to compute $C:=C-A B\left(C \in \mathbb{R}^{m \times n}, A \in \mathbb{R}^{m \times k}\right.$, and $B \in \mathbb{R}^{k \times n}$ ) attains the best performance when $k=128$. Notice that most computation in the SA LINPACK procedure is cast in terms of this operation, with $k=b$.

The performance benefits reported on this platform are representative of the benefits that can be expected on other current architectures.

\subsection{Results}

In Figure 8(top) we show the speedup attained when an existing factorization of $B$ is reused by reporting the time required to factor (1) with the high-performance LU factorization with partial pivoting divided by the time required to update an existing factorization of $B$ via the SA LINPACK procedure (Steps 2-5). In that figure, $n_{B}=1000$ and $n_{E}$ is varied from 0 to 1000 . The results are reported when different block sizes $b$ are chosen. The DGEMM operation, in terms of which most computation is cast, attains the best performance when $b=128$ is chosen. However, this generates enough additional flops that the speedup is better when $b$ is chosen to be smaller. When $n_{E}$ is very small, $b=8$ yields the best performance. As $n_{E}$ increases performance improves by choosing $b=32$.

The effect of the overhead of the extra computations is demonstrated in Figure 8(bottom). There, we report the ratio of the time required by Steps 1-5 of the SA LINPACK procedure divided by the time required by the LU factorization with partial pivoting of (1).

\section{Conclusions}

We have proposed blocked algorithms for updating an LU factorization. They have been shown to attain high performance and to greatly reduce the cost of an update to a matrix for which a partial factorization already exists. The key insight is the synthesis of LINPACK- and LAPACK-style pivoting. While some additional computation is required, this is more than offset by the improvement in performance that comes from casting computation in terms of matrix-matrix multiplication. 

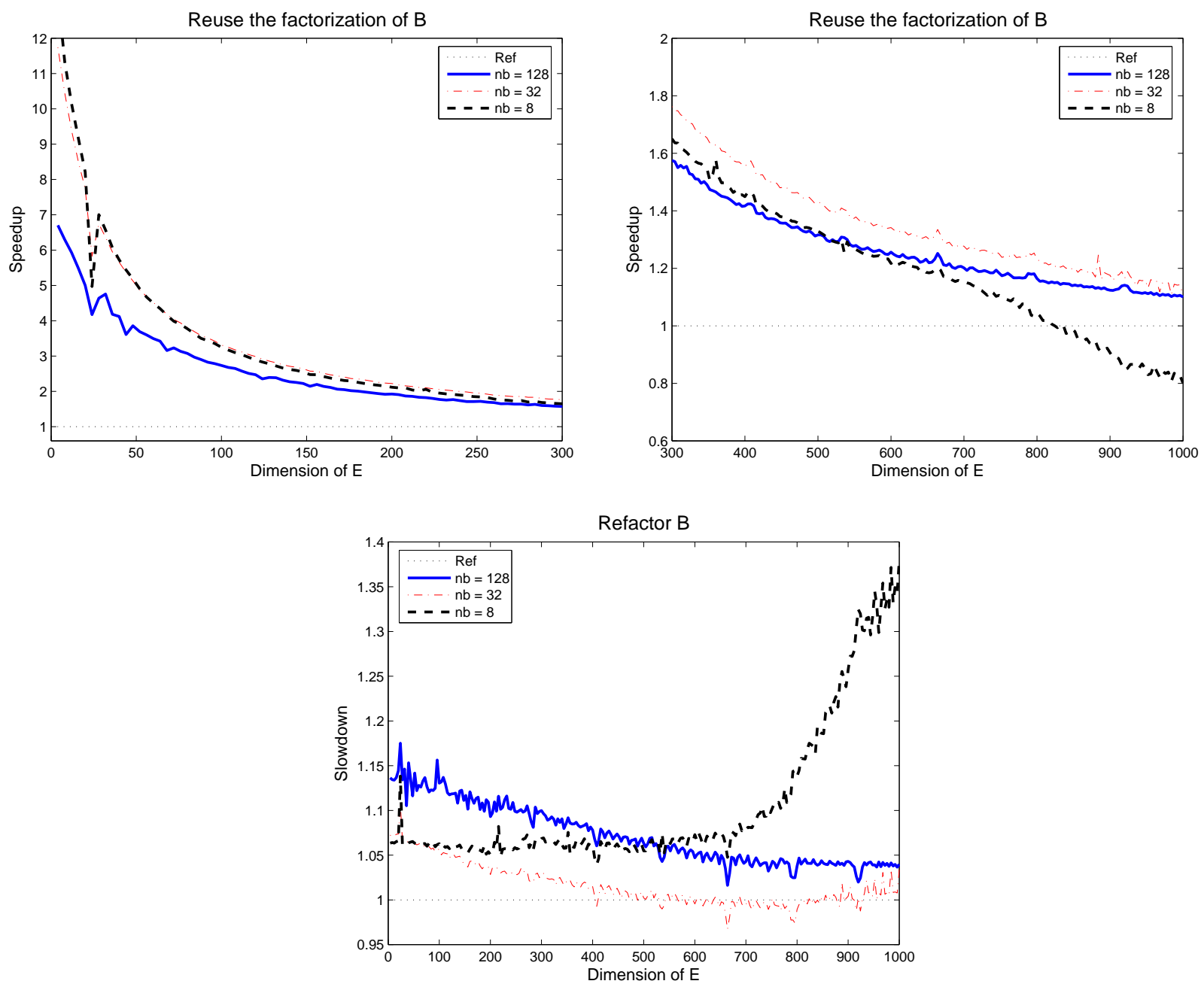

Figure 8: Top: Speedup attained when $B$ is not refactored, over LU factorization with partial pivoting of the entire matrix. Bottom: Slowdown for the first factorization (when $B$ must also be factored).

\section{Acknowledgments}

This research was partially sponsored by NSF grants ACI-0305163, CCF-0342369 and CCF-0540926, and an equipment donation from Hewlett-Packard. Primary support for this work came from the J. Tinsley Oden Faculty Fellowship Research Program of the Institute for Computational Engineering and Sciences (ICES) at UT-Austin.

For further information on FLAME visit www.cs.utexas.edu/users/flame.

\section{References}

[1] Paolo Bientinesi, John A. Gunnels, Margaret E. Myers, Enrique S. Quintana-Ortí, and Robert A. van de Geijn. The science of deriving dense linear algebra algorithms. ACM Trans. Math. Soft., 31(1):1-26, March 2005. 
[2] Paolo Bientinesi and Robert van de Geijn. Representing dense linear algebra algorithms: A farewell to indices. Technical Report FLAME Working Note 17, CS-TR-2006-10, Department of Computer Sciences, The University of Texas at Austin, 2006.

[3] Tom Cwik, Robert van de Geijn, and Jean Patterson. The application of parallel computation to integral equation models of electromagnetic scattering. Journal of the Optical Society of America A, 11(4):1538-1545, April 1994.

[4] Jim Demmel and Jack Dongarra. LAPACK 2005 prospectus: Reliable and scalable software for linear algebra computations on high end computers. LAPACK Working Note 164 UT-CS-05-546, University of Tennessee, February 2005.

[5] Po Geng, J. Tinsley Oden, and Robert van de Geijn. Massively parallel computation for acoustical scattering problems using boundary element methods. Journal of Sound and Vibration, 191(1):145-165, 1996.

[6] K. Goto and R. van de Geijn. Anatomy of high-performance matrix multiplication. ACM Trans. Math. Soft., 2006. Submitted.

[7] Kazushige Goto. http://www.tacc.utexas.edu/resources/software/, 2006.

[8] John A. Gunnels, Greg M. Henry, and Robert A. van de Geijn. A family of high-performance matrix multiplication algorithms. In Vassil N. Alexandrov, Jack J. Dongarra, Benjoe A. Juliano, René S. Renner, and C.J. Kenneth Tan, editors, Computational Science - ICCS 2001, Part I, Lecture Notes in Computer Science 2073, pages 51-60. Springer-Verlag, 2001.

[9] Brian Gunter and Robert van de Geijn. Parallel out-of-core computation and updating of the QR factorization. ACM Trans. Math. Soft., 31(1):60-78, March 2005.

[10] F. Gustavson, A. Henriksson, I. Jonsson, B. Kågström, and P. Ling. Superscalar GEMM-based level 3 BLAS - the on-going evolution of a portable and high-performance library. In B. Kågström et al., editor, Applied Parallel Computing, Large Scale Scientific and Industrial Problems, Lecture Notes in Computer Science 1541, pages 207-215. Springer-Verlag, 1998.

[11] Nicholas J. Higham. Accuracy and Stability of Numerical Algorithms. Society for Industrial and Applied Mathematics, Philadelphia, PA, USA, second edition, 2002.

[12] Thierry Joffrain, Enrique S. Quintana-Ortí, and Robert A. van de Geijn. Rapid development of highperformance out-of-core solvers. In J. Dongarra, K. Madson, and J. Wasńiewski, editors, PARA 2004, LNCS 3732, pages 413-422. Springer-Verlag, 2005.

[13] B. Kågström, P. Ling, and C. Van Loan. Gemm-based level 3 blas: High-performance model, implementations and performance evaluation benchmark. LAPACK Working Note \#107 CS-95-315, Univ. of Tennessee, Nov. 1995.

[14] B. Kågström, P. Ling, and C. Van Loan. GEMM-based level 3 BLAS: High performance model implementations and performance evaluation benchmark. ACM Trans. Math. Soft., 24(3):268-302, 1998.

[15] Ken Klimkowski and Robert van de Geijn. Anatomy of an out-of-core dense linear solver. In Proceedings of the International Conference on Parallel Processing 1995, volume III - Algorithms and Applications, pages 29-33, 1995.

[16] Danny C. Sorensen. Analysis of pairwise pivoting in Gaussian elimination. IEEE Trans. on Computers, c-34(3):274-278, 1985.

[17] G. W. Stewart. Matrix Algorithms. Volume I: Basic Decompositions. SIAM, Philadelphia, 1998. 
[18] S. Toledo and F. Gustavson. The design and implementation of SOLAR, a portable library for scalable out-of-core linear algebra computations. In Proceedings of the fourth workshop on I/O in parallel and distributed systems, pages 28-40, 1996.

[19] Sivan Toledo. A survey of out-of-core algorithms in numerical linear algebra. In James Abello and Jeffrey Scott Vitter, editors, External Memory Algorithms and Visualization, pages 161-180. American Mathematical Society Press, Providence, RI, 1999.

[20] Lloyd N. Trefethen and Robert S. Schreiber. Average-case stability of Gaussian elimination. SIAM J. Matrix Anal. Appl., 11(3):335-360, 1990.

[21] J. H. Wilkinson. The Algebraic Eigenvalue Problem. Oxford University Press, London, 1965.

[22] E. Yip. Fortran subroutines for Out-of-Core solutions of linear systems. Technical Report CR-158142, NASA, 1979. 